EPJ manuscript No.

(will be inserted by the editor)

\title{
A NEW APPLICATION OF THE GÜRSEY AND RADICATI MASS FORMULA
}

\author{
M.M. Giannini ${ }^{1}$, E. Santopinto ${ }^{1}$, and A.Vassallo ${ }^{1}$ \\ Dipartimento di Fisica, Università degli Studi di Genova \& I.N.F.N. Sezione di Genova
}

July 27,2021

\begin{abstract}
We study the spin- and flavour- dependent $S U(6)$ violations in the baryon spectrum by means of a Gürsey Radicati mass formula. The average energy of each $S U(6)$-multiplet is described using the $S U(6)$ invariant interaction given by a hypercentral potential containing a linear and a hypercoulomb term. We show that the non strange and strange baryon masses are in general fairly well reproduced and moreover that the Gürsey Radicati formula holds in a satisfactory way also for the excited states up to $2 \mathrm{GeV}$. The coefficients of the Gürsey Radicati $S U(6)$ breaking part obtained by the fit of the three-quark spectrum can be used to evaluate in first approximation the splitting within multiplets also for exotic baryon systems.
\end{abstract}

PACS. 12.39-x Phenomenological quark models -12.39 .Pn Quark potential models $-11.30 . \mathrm{Hv}$ Flavor symmetries in particles and fields - 11.30.Ly Other internal and higher symmetries in particles and fields

\section{Introduction}

Different versions of Constituent Quark Models (CQM) have been proposed in the last decades in order to describe the baryon properties. What they have in common is the fact that the threequark interaction can be separated in two parts: the first one, containing the confinement interaction, is spin and flavour independent and it is therefore $S U(6)$ invariant, while the second violates the $S U(6)$ symmetry. This separation has been supported by the very first Lattice QCD calculations [1] and is confirmed by the most recent ones [2 3]. The various CQMs differ in the way the $S U(6)$ invariance is violated. One of the most popular ways was the introduction of a hyperfine (spinspin) interaction 4567], however in many studies a spinand isospin- [8910 11] or a spin- and flavour-dependent interaction 8910 has been considered. In this paper we study the symmetries of the baryon spectrum using a very simple approach based on the Gürsey Radicati mass formula (GR) [12]. It is well known that the baryon spectrum exhibits an approximated $S U(6)$ symmetry and that the GR mass formula, despite it's simplicity, describes quite well the way this symmetry is broken, at least in the lower part of the baryon spectrum. Our idea is to build up a very simple model based on the GR mass formula, to fix the parameters of the model in way to obtain the best description (within the limits of this approach) of the baryon spectrum and thereafter use the model (with the parameters values fixed on the baryon spectrum) to give predictions for the masses of other hadronic systems as for example pentaquarks [13]. The model we propose is a simple CQM where the $S U(6)$ invariant part of the Hamiltonian is the same as in the Hypercentral Constituent Quark Model [6 7] and where the $S U(6)$ symmetry is broken by a GR inspired interaction.

In the second section we briefly remind the hypercentral CQM and its results, then in the third section we construct the model using the GR as the $S U(6)$ breaking term. In the fourth section we give the results obtained by fitting the GR parameters to the strange and non strange baryon energies and we compare the spectrum with the experimental data. Finally we discuss our results.

\section{The hypercentral model}

The experimental 4- and 3-star non strange resonances can be arranged in $S U(6)$ multiplets. This means that the quark dynamics has a dominant $S U(6)$ invariant part, which accounts for the average multiplet energies. In the Hypercentral Constituent Quark Model (hCQM) it is assumed to be given by the hypercentral potential [6]

$$
V(x)=-\frac{\tau}{x}+\alpha x
$$

where

$$
x=\sqrt{\rho^{2}+\lambda^{2}},
$$

is the hyperradius and $\rho$ and $\boldsymbol{\lambda}$ are the Jacobi coordinates describing the internal quark motion. Interactions of the type linear plus Coulomb-like have been used since long time for the meson sector, e.g. the Cornell potential. This form has also been obtained in recent Lattice QCD calculations [2 3] for $S U(3)$ invariant static quark sources. Introducing, along with the hypercentral potential, a standard hyperfine interaction [4] which breaks the $S U(6)$ symmetry, the hCQM has given a fair description of the non strange baryon spectrum [6] and of other various physical quantities of interest: the photocouplings [14], 
the electromagnetic transition amplitudes [15], the elastic nucleon form factors $[16]$ and the ratio between the electric and magnetic proton form factors [17].

Subsequently, in order to improve the description of the non strange spectrum, an isospin dependent $S U(6)$ violating term has been introduced [11]. The complete interaction used in this latter case is given by

$$
H_{\text {int }}=V(x)+H_{\mathrm{S}}+H_{\mathrm{I}}+H_{\mathrm{SI}},
$$

where $V(x)$ is the linear plus hypercoulomb SU(6)-invariant potential of eq. (1), while $H_{\mathrm{S}}$ is the hyperfine interaction and $H_{\mathrm{I}}, H_{\mathrm{SI}}$ are respectively isospin and spin-isospin dependent terms. Similar results can be obtained in a relativized version of the model [18], in which the quark kinetic energy has the correct relativistic form.

The preceding results show that both spin and isospin (or flavour) dependent terms in the quark Hamiltonian are important. Their contributions can be considered as perturbative $S U(6)$-violating terms added to the unperturbed $S U(6)$-invariant energies provided by the hypercentral potential of eq. (1).

\section{The strange baryon spectrum and the Gürsey Radicati mass formula}

The spin and isospin dependent interactions considered in the previous section are not the only source of $S U(6)$ violation. In order to study the strange baryon spectrum one has to consider the $S U(3)$ violation produced by the differences in the quark masses. The well known Gell-Mann-Okubo (GMO) mass formula [19] made use of a $\lambda_{8}$ violation of $S U(3)$ in order to describe the mass splittings within the various $S U(3)$ multiplets; according to this formula the mass $M$ of a baryon belonging to a given $S U(3)$ multiplet can be expressed as

$$
M=M_{0}+D Y+E\left[T(T+1)-\frac{1}{4} Y^{2}\right],
$$

where $M_{0}$ is the average energy value of the $S U(3)$ multiplet, $Y$ is the hypercharge, $T$ is the Isospin of the baryon and $D$ and $E$ are parameters to be fitted. A simple way to interpret the origin of such a violation is just to attribute to the strange quark a mass different from the up and down quark ones. The calculations were performed without reference to any explicit dynamical model, but using standard group theoretical methods. The unknown parameters $D$ and $E$ in the $S U(3)$ violating terms can be in principle fitted to the experimental masses, thereby providing a phenomenological way to describe the spectrum.

A similar approach for the description of the splittings within the SU(6) baryon multiplets is provided by the Gürsey Radicati mass formula [12]. In the original paper the mass formula reads:

$$
M=M_{0}+C S(S+1)+D Y+E\left[T(T+1)-\frac{1}{4} Y^{2}\right]
$$

where $S$ is the spin. Eq. (5) can be rewritten in terms of Casimir operators in the following way

$$
\begin{aligned}
M= & M_{0}+C C_{2}\left[S U_{S}(2)\right]+D C_{1}\left[U_{Y}(1)\right] \\
& +E\left[C_{2}\left[S U_{I}(2)\right]-\frac{1}{4}\left(C_{1}\left[U_{Y}(1)\right]\right)^{2}\right]
\end{aligned}
$$

where $C_{2}\left[S U_{S}(2)\right]$ and $C_{2}\left[S U_{I}(2)\right]$ are the $S U(2)$ (quadratic) Casimir operators for spin and isospin, respectively, and $C_{1}\left[U_{Y}(1)\right]$ is the Casimir for the $U(1)$ subgroup generated by the hypercharge $Y$. The presence of a spin dependent term is necessary since states belonging to a definite $S U(6)$ multiplet do not have the same spin value. This mass formula has proven to be successful in the description of the gruond state baryon masses, however, as stated by the authors themselves, eq. 66 is not the most general mass formula that can be written on the basis of a broken $S U(6)$ symmetry.

In order to generalize eq. (6) one can consider a dynamical spin-flavour symmetry $S U_{S F}(6)$ and write the following chain of subgroups

$$
\begin{array}{cccccc}
S U_{S F}(6) & \supset S U_{F}(3) \otimes S U_{S}(2) & \supset S U_{I}(2) \otimes U_{Y}(1) \otimes S O_{S}(2) \\
\downarrow & \downarrow & \downarrow & \downarrow & \downarrow & \downarrow \\
\left(\lambda_{1}, . . \lambda_{5}\right) & \left(\lambda_{f}, \mu_{f}\right) & S & I & Y & M_{S}
\end{array}
$$

where in the bottom row we report the quantum numbers which label the irreducible representations of the corresponding groups. Therefore one can describe the $S U_{S F}(6)$ symmetry breaking mechanism by generalizing eq. (6) as

$$
\begin{array}{r}
M=M_{0}+A C_{2}\left[S U_{S F}(6)\right]+B C_{2}\left[S U_{F}(3)\right] \\
+C C_{2}\left[S U_{S}(2)\right]+D C_{1}\left[U_{Y}(1)\right] \\
+E\left(C_{2}\left[S U_{I}(2)\right]-\frac{1}{4}\left(C_{1}\left[U_{Y}(1)\right]\right)^{2}\right)
\end{array}
$$

The generalized Gürsey Radicati mass formula eq. [8 can be used to describe the whole baryon spectrum, provided that two conditions are fulfilled. The first condition is the possibility of using the same splitting coefficients for different $S U(6)$ multiplets. This seems actually to be the case, as shown by the algebraic approach to the baryon spectrum [8], where a formula similar to eq. 8 has been applied. The second condition is given by the possibility of getting reliable values for the unperturbed mass values $M_{0}$. Our idea is to use for this purpose the $S U(6)$ invariant part of the hCQM, which provides a good description of the non strange baryon spectrum and to introduce a Gürsey Radicati inspired $S U(6)$ breaking interaction to describe the splittings within each $S U(6)$ multiplet. We shall therefore make use of the following three quark Hamiltonian

$$
H=H_{0}+H_{G R}
$$

with

$$
H_{0}=3 m+\frac{\boldsymbol{p}_{\lambda}^{2}}{2 m}+\frac{\boldsymbol{p}_{\rho}^{2}}{2 m}+V(x)
$$

and

$$
\begin{gathered}
H_{G R}=+A C_{2}\left[S U_{S F}(6)\right]+B C_{2}\left[S U_{F}(3)\right]+C C_{2}\left[S U_{S}(2)\right] \\
+D C_{1}\left[U_{Y}(1)\right]+E\left(C_{2}\left[S U_{I}(2)\right]-\frac{1}{4}\left(C_{1}\left[U_{Y}(1)\right]\right)^{2}\right)
\end{gathered}
$$

where $V(x)$ is the hypercentral potential of eq.11, and $\mathrm{m}$ is the constituent quark mass. It has to be remarked that, in order to simplify the solving procedure, the constituent quark masses are assumed to be the same for all the quark flavours ( $\left.m_{u}=m_{d}=m_{s}=m\right)$, therefore, within this approximation, the $S U(3)$ symmetry is only broken dynamically by the 
spin and flavour dependent terms in the Hamiltonian. In other words, in this approximation, the effects of the strange quark mass on the baryon spectrum are described by the two terms of eq.(4).

The eigenproblem of $H_{0}$ can be solved numerically, the spinflavour part of the resulting eigenfunctions has definite properties under transformations of the $S U_{S F}(6)$ group and its subgroups. Using the notation of equation (7) the spin-flavour part of the wave function can be written as

$$
\left|\left(\lambda_{1}, \lambda_{2}, \lambda_{3}, \lambda_{4}, \lambda_{5}\right),\left(\lambda_{f}, \mu_{f}\right), I, Y, S, M_{S}\right\rangle .
$$

Often the irreducible representations are identified not by the quantum numbers but by their dimension. Thus, for example

$$
\begin{gathered}
\left|(3,0,0,0,0),(2,1), I=\frac{1}{2}, Y=1, S=\frac{1}{2}, M_{S}\right\rangle \\
\left.\left.\equiv\right|^{2} 8_{1 / 2},\left[56,0^{+}\right], N\right\rangle
\end{gathered}
$$

is the spin-flavour part of the nucleon wave function. The notation used is

$$
\left.\left.\right|^{2 S+1} \operatorname{dim}(S U(3))_{J},\left[\operatorname{dim}(S U(6)), L^{P}\right], X\right\rangle,
$$

where $\operatorname{dim}(S U(n))$ is the dimension of the $S U(n)$ representation, $S$ and $L$ are the total spin and orbital angular momentum of the quark system, respectively, $J$ and $P$ are the spin and parity of the resonance and $X=N, \Delta, \ldots$ denotes the type of baryon resonance.

The action of $H_{G R}$ on the eigenstates of $H_{0}$ is completely identified by the expectation values of the Casimir operators on the states of eq. 10

$$
\begin{aligned}
\left\langle C_{2}\left[S U_{S F}(6)\right]\right\rangle & =\left\{\begin{array}{l}
45 / 4 \text { for }[56] \\
33 / 4 \text { for }[70] \\
21 / 4 \text { for }[20]
\end{array}\right. \\
\left\langle C_{2}\left[S U_{F}(3)\right]\right\rangle & =\left\{\begin{array}{l}
3 \text { for }[8] \\
6 \text { for }[10] \\
0 \text { for }[1]
\end{array}\right. \\
\left\langle C_{2}\left[S U_{I}(2)\right]\right\rangle & =I(I+1) \\
\left\langle C_{1}\left[U_{Y}(1)\right]\right\rangle & =Y \\
\left\langle C_{2}\left[S U_{S}(2)\right]\right\rangle & =S(S+1)
\end{aligned}
$$

Therefore the mass of each baryon state $|B\rangle$ can be written as:

$$
\langle B|H| B\rangle=E_{\gamma \nu}+\left\langle B\left|H_{G R}\right| B\right\rangle
$$

where $E_{\gamma \nu}$ denotes the eigenvalue of $H_{0}$ with $\gamma=2 n+l_{\rho}+l_{\lambda}$ ( $n$ being a non negative integer), $\nu$ denotes the number of nodes of the space three quark wave functions and $l_{\rho}, l_{\lambda}$ are the orbital angular momenta corresponding to the Jacobi coordinates (see e.g. [6]).

Since $H_{G . R}$. does not depend on the spatial degrees of freedom, the $S U(6)$ breaking term introduced in this model is diagonal in the baryon states, this means that the Gürsey Radicati term is able to give energy splittings within the $S U(6)$ multiplets, but no configuration mixing effects can arise from such an interaction ${ }^{1}$. Therefore the model is expected to fail in the description

1 The kind of problems that one can face neglecting the spatial dependence on the $S U(6)$ breaking part is discussed by Jennings and Maltman [20] of all thoose observables where a good description of the three quark wave function is crucial.

\section{Results}

The parameters in $H_{G R}$ can be determined in order to reproduce the experimental values of the energy splittings. We first adopt an analytical procedure by means of which we choose a limited number of well known resonances and express their mass differences using $H_{G R}$ and the Casimir operator expectation values given in the previous section. We list in the following the analytical expressions for the mass differences of the chosen pairs of resonances:

$$
\begin{aligned}
(N(1650) S 11-N(1535) S 11) & =3 C \\
(\Delta(1232) P 33-N(938) P 11) & =9 B+3 C+3 E \\
(N(1535) S 11-N(1440) P 11) & =\left(E_{10}-E_{01}\right)+12 A \\
(\Sigma(1193) P 11-N(938) P 11) & =\frac{3}{2} E-D \\
(\Lambda(1116) P 01-N(938) P 11) & =-D-\frac{1}{2} E .
\end{aligned}
$$

Looking at eq. 13 it is easy to understand that for the description of the non-strange baryon spectrum the only relevant parameters are $A, C$ and the combination $(3 B+E)$. It should be noted that, in order to apply the Gürsey Radicati mass formula to the excited states, it is necessary to know the coefficient $A$ of the $S U(6)$ Casimir operator and the excited energies provided by the CQM.

Once the SU(6) breaking interaction has been determined, the parameters of $H_{0}$ ( $\alpha$ and $\tau$ ) which lead to the unperturbed energies $E_{\gamma \nu}$ can be fixed by a minimization procedure on the non-strange baryon spectrum. The complete list of the parameter values is reported in Table 1 column (I). In this way the $E_{\gamma \nu}$ levels are placed close to the central mass value of each SU(6) multiplet. As shown in eq. 13, a further adjustment to the unperturbed multiplet energy is provided by the presence of the $S U(6)$ Casimir operator. The resulting spectrum is shown in Fig. 11 and Table 2 column $M_{\text {calc }}^{I}$. Despite of the simple form of the SU(6) breaking interaction, the general features of the spectrum are fairly well reproduced, especially in the low energy part. It has to be noted in particular that, besides the ground state masses which have been fixed through eq.13, the predicted masses of the $\Sigma^{*}, \Xi, \Xi^{*}$ and $\Omega$ states are nicely close to the experimental values.

The second approach followed in the application of the Gürsey Radicati mass formula is to fit all parameters at the same time in order to obtain the best reproduction of the spectrum of the 3 and 4 star resonances ${ }^{2}$. The fitted parameters are reported in Table 1 column (II), while the resulting spectrum is shown in Fig. (2) and the corresponding numerical values are given in Table 2 column $M_{\text {calc. }}^{I I}$. The result is a better overall agreement with the experimental data, even if the prediction in the non strange sector is worsened. For this reason, we prefer the

\footnotetext{
2 The PDG [21] quotes a three stars $\Xi(1690)$ resonance; however, since the values of spin and parity are not known, this resonance cannot be identified with a definite eigenstate of the Hamiltonian and therefore this state has been excluded from our analysis.
} 
values of the parameters obtained with the previous analytical method since we have used only well known and well established resonances in order to fix the parameters.

In both cases a non zero value of the $S U(6)$ Casimir coefficient is needed in order to reproduce the average multiplet energies. We have also tried a fit imposing $A=0$. The resulting parameters $\alpha$ and $\tau$ are however considerably different with respect to those of Table 2 because the lack of the parameter $A$ must be compensated by the $S U(6)$ invariant energies provided by the hypercentral potential. This is particularly evident in the case of the negative parity resonances, where the energy difference $E_{10}-E_{01}$ must be bigger than in the previous case in order to obtain a good reproduction of the masses; in this way, however, the right ordering of the Roper resonance and the negative parity resonances is lost. This means that the presence of the Casimir $C_{2}\left[S U_{S F}(6)\right]$ is needed and its effect is to shift down the energy of the first excited $0^{+}$state with respect to that of the $1^{-}$. The effect of this term is very similar to that of the phenomenological $U$ potential of the Isgur-Karl model [4]

The mass formula of eq. (8) can be used to add a simple SU(6) breaking interaction to a CQM and despite its simplicity it gives rise to a good description of the baryon spectrum. Of course for the wavefunctions, and other observables, it is not expected to be as successful as for the spectrum.

Another important feature of this kind of approach is the model independence of the $S U(6)$ breaking part of the Hamiltonian. Looking at eq.113 it is easy to understand that the values of the parameters of the $\mathrm{SU}(6)$ breaking part of the Hamiltonian (i.e. the B,C,D,E parameters) are completely independent on the choice of $H_{0}+A C_{2}\left[S U_{S F}(6)\right]$ which must describe the central mass value of each $S U(6)$ multiplet. An important consequence of the independence, in first approximation, of the coefficients of the Casimir operators on the particular wave functions, is the possibility of using the same $S U(6)$ breaking Hamiltonian for systems with a different number of quarks. This has been done in a recent study of the pentaquark spectrum, where the Gürsey Radicati mass formula of eq. 8 has been used for a systematic analysis of the $(S U(6)$ breaking) splittings within the exotic baryon multiplets [13].

Finally, we present some comments on the Gürsey Radicati mass formula of eq. [8]. As we have already observed, the last two terms, that is those with coefficients $D$ and $E$, describe up to first order the $S U(6)$-violation coming from the mass difference of quarks, as it has been done in the Gell-Mann-Okubo formula. The remaining terms are expected to appear once an explicit dynamics for the quark system is introduced. For example, in a recent calculation of multiquark state energies, a spin-flavour dependent interaction of the type

$$
H_{\lambda \sigma}=\sum_{i<j}^{n}\left(\boldsymbol{\lambda}_{i} \cdot \boldsymbol{\lambda}_{j}\right)\left(\boldsymbol{\sigma}_{i} \cdot \boldsymbol{\sigma}_{j}\right)
$$

has been introduced [22]; $\lambda_{i}$ are the $S U_{\text {flavour }}(3)$ matrices. The matrix elements of such spin-flavour interaction between states belonging to definite irreducible representations of $S U(6)$, $S U(3)_{\text {flavour }}$ and $S U(2)_{\text {spin }}$ can be calculated as

$$
\begin{aligned}
& \left\langle[f]^{S U(6)}[f]^{S U(3)}[f]^{S U(2)}\left|H_{\lambda \sigma}\right|[f]^{S U(6)}[f]^{S U(3)}[f]^{S U(2)}\right\rangle \\
& =4 C_{2}(S U(6))-2 C_{2}(S U(3))-\frac{4}{3} C_{2}(S U(2))-8 N_{q}
\end{aligned}
$$

where $N_{q}$ is the number of quarks [22] and $C_{2}(S U(2))$ is given by $S(S+1), S$ being the total spin. If the spatial dependence of the $S U(6)$ breaking terms is not neglected, this is no more true.

As a conclusion, we can say that the Gürsey Radicati 87 is a simple way to parametrize at the first order the possible $S U(6)$ breaking terms of the strong interaction. The approach we have adopted here is then to parametrize all the $S U(6)$-breaking terms by means of the generalized Gürsey Radicati, without formulating any hypothesis on the microscopic mechanism (onegluon exchange, Goldstone-boson interaction, chiral soliton ...). The next step will be to introduce an explicit $S U(6)$ breaking term, containing also the spatial dependence and with a clear microscopic origin.

\section{Discussion}

We have shown that the Gürsey Radicati (GR) mass formula is a good parametrization of the baryon energy splittings coming from $S U(6)$ breaking. The splittings are considered as perturbations superimposed to the $S U(6)$ invariant levels, which, in our approach, are given by the hypercentral three-quark potential [6]. The overall good description of the spectrum which we obtain shows that the GR mass formula can also be used to give a fair description of the energies of the excited multiplets at least up to $2 \mathrm{GeV}$ and not only for the ground state octets and decuplets, where it has been originally applied. There are still problems with the reproduction of some hyperons, in particular for the $\Lambda(1405)$ and the $\Lambda(1520)$ resonances that come out degenerate and above the experimental values. There are problems in the reproduction of the experimental masses also in the $\Sigma$ sector where both the $\Sigma(1670) D 13$ and the $\Sigma(1775) D 15$ four stars resonances turn out to have predicted masses about $100 \mathrm{MeV}$ above the experimental values. A better agreement can be obtained either using the square of the mass [8] or trying to include a spatial dependence in the $S U(6)$-breaking part, which may have, among others, a delta or Gaussian factor, in order to decrease the breaking with the increase of the spatial excitation. Although the space dependence of the $S U(6)$ breaking terms has to be neglected in order to apply the GR formula, We can consider the Gürsey Radicati $S U(6)$ breaking as the first order parametrization of the splittings due to an interaction which depends also on the coordinates. Within this approximation it can be used for the description of the $\mathrm{SU}(6)$-breaking effects independently from the way in which one describes the spatial part. It can be applied, with the same coefficients, also to systems with different number of quarks, such as baryons or pentaquarks. A similar statement is valid if we restrict ourselves to $S U(3)$ breaking, using a Gell-MannOkubo mass formula. In fact, the representations involved will differ for each system and the dependence on the number of quarks will be accounted for by the different values of the various Casimir operators. On the contrary, the unperturbed $S U(6)$ invariant levels will depend on the number of quarks and on the way in which the spatial part is described, that is an explicit dynamics must be considered.

What we have presented here is not the only example of such a situation. In fact recently in [23] a Hamiltonian, containing 
the quadratic $S U(3)$ Casimir and a Gell-Mann Okubo symmetry breaking term, has been used for the calculations of energy splittings both for baryons and pentaquarks. This is an indication that different effective models for the baryons at the first oder give origin to a Gell-Mann Okubo mass formula, independently from the fact that we consider a Chiral Soliton Model or a bag model or a CQM, that means independently from which effective degrees of freedom we use and how we describe from a spatial point of view the baryon bound states.

\section{References}

1. A. De Rjula, H. Georgi and S.L. Glashow, Phys. Rev. D12, 147 (1975)

2. Gunnar S. Bali et al., Phys. Rev. D62, 054503 (2000); Gunnar S. Bali, Phys. Rep. 343, 1 (2001).

3. C. Alexandrou, P. de Forcrand and O. Jahn, Nucl. Phys. Proc. Suppl. 119, 667 (2003); H. Suganuma, T. T. Takahashi, F. Okiharu and H. Ichie, hep-lat/0407014

4. N. Isgur and G. Karl, Phys. Rev. D18, 4187 (1978); Phys. Rev. D19, 2653 (1979).

5. S. Godfrey and N. Isgur, Phys. Rev. D32, 189 (1985).

6. M. Ferraris, M.M. Giannini, M. Pizzo, E. Santopinto and L. Tiator, Phys. Lett. B364, 231 (1995).

7. E. Santopinto, F. Iachello and M. M. Giannini, Eur. Phys. J. A 1 (1998) 307.

8. R. Bijker, F. Iachello and A. Leviatan, Ann. Phys. (N.Y.) 236, 69 ( 1994)

9. L. Ya. Glozman and D.O. Riska, Phys. Rep. C268, 263 (1996).

10. L. Ya. Glozman, Z. Papp, W. Plessas, K. Varga, R. F. Wagenbrunn, Phys. Rev. C57, 3406 (1998); L. Ya. Glozman, W. Plessas, K. Varga, R. F. Wagenbrunn, Phys. Rev. D58, 094030 (1998).

11. M.M. Giannini, E. Santopinto and A, Vassallo, Eur. Phys. J. A12, 447 (2001).

12. F. Gürsey and L.A. Radicati, Phys. Rev. Lett. 13, 173 (1964).

13. R. Bijker, M.M. Giannini and E. Santopinto, hep-ph/0310281 Eur. Phys. J. A22, 319 (2004).

14. M. Aiello, M. Ferraris, M.M. Giannini, M. Pizzo and E. Santopinto, Phys. Lett. B387, 215 (1996).

15. M. Aiello, M. M. Giannini, E. Santopinto, J. Phys. G: Nucl. Part. Phys. 24, 753 (1998)

16. M. De Sanctis, E. Santopinto, M.M. Giannini, Eur. Phys. J. A1, 187 (1998).

17. M. De Sanctis, M.M. Giannini, L. Repetto, E. Santopinto, Phys. Rev. C62,025208 (2000).

18. M.M. Giannini, E. Santopinto, M. Traini and A. Vassallo, to be published.

19. See e.g. M. Gell-Mann and Y. Ne'eman, The eightfold way (W.A. Benjamin, Inc., New York, 1964).

20. B. K. Jennings and K. Maltman, Phys. Rev. D 69, 094020 (2004).

21. Particle Data Group, Eur. Phys. J. C15, 1 (2000).

22. C. Helminen and D.O. Riska, Nucl. Phys. A 699, 624 (2002).

23. D. Diakonov, V. Petrov and M. Polyakov, Z. Phys. A 359, 305 (1997). 

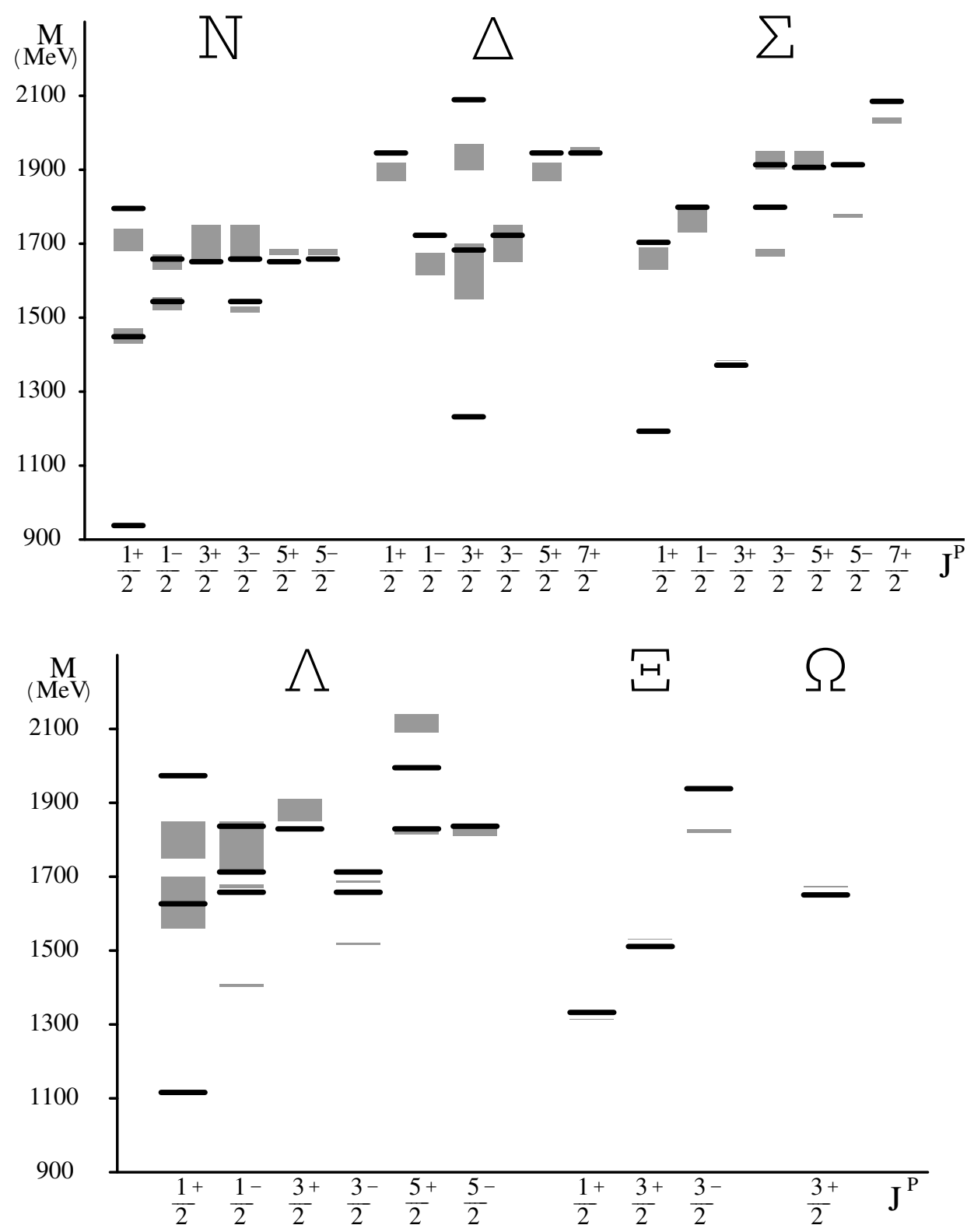

Fig. 1. The energy levels (black lines) for the 3 and 4 star resonances obtained with the Hamiltonian 9 fixing the parameters as described in equation 13. The numerical values of the calculated masses of baryon resonances are reported in Table 2 column $M_{\text {calc }}^{I}$. The values of the parameters of the Hamiltonian are reported in column (I) of Table 1 The experimental data are taken from PDG [21] (gray boxes). 

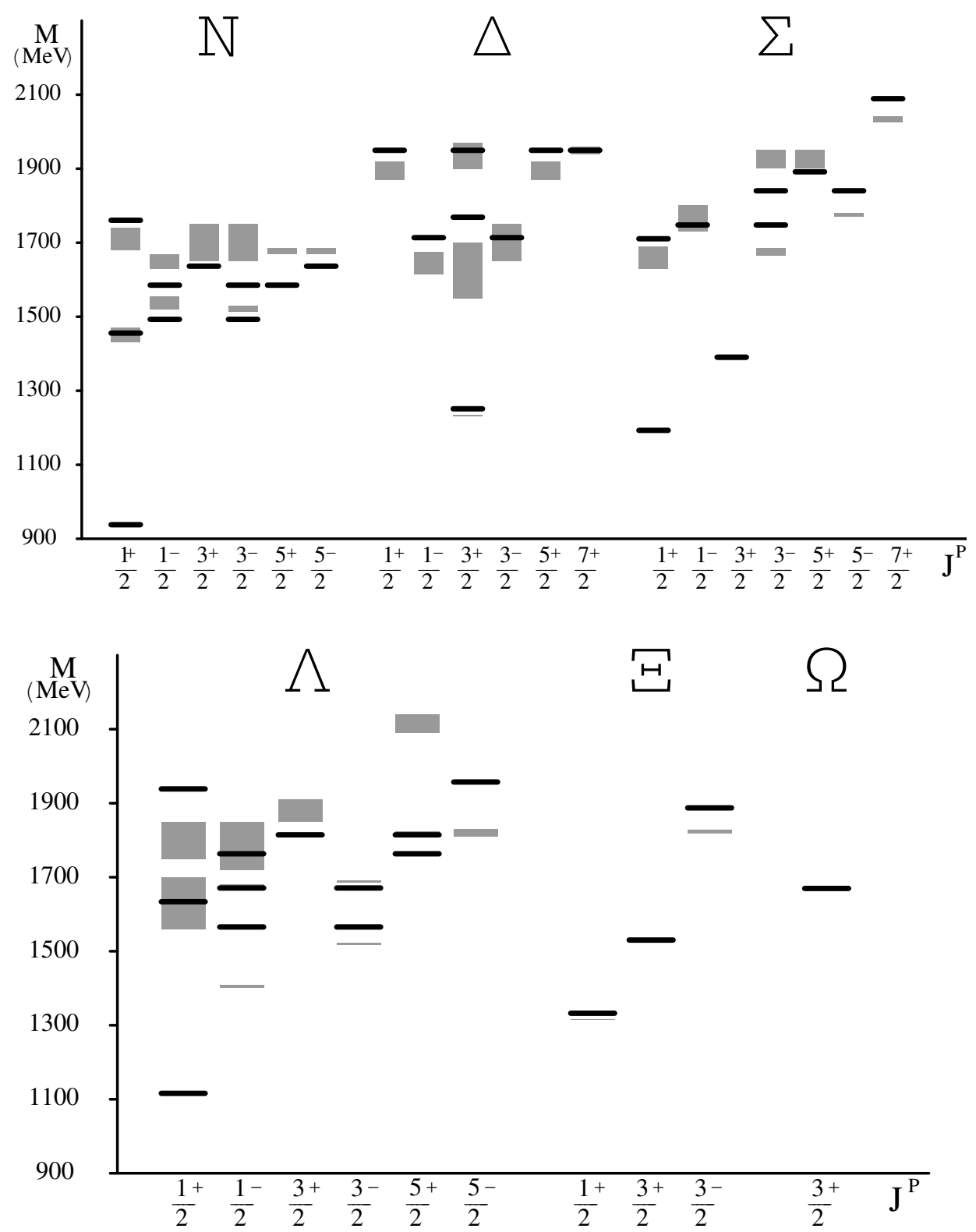

Fig. 2. The energy levels (black lines) for the 3 and 4 star resonances obtained with the Hamiltonian 9 fixing the Hamiltonian parameters by a fitting procedure. The resulting values of the parameters are reported in column II of Table 1 while the numerical values of the calculated masses are also reported in Table 2 column $M_{\text {calc }}^{I I}$. The experimental data are taken from PDG [21] (gray boxes). 
Table 1. The fitted values of the parameters of the Hamiltonian 9 . Column (I) corresponds to the analytical fixing procedure of eq. 13, while column (II) contains the values obtained with a global fit to the experimental resonance masses.

\begin{tabular}{crr}
\hline Parameter & (I) & (II) \\
\hline$\alpha=$ & $1.4 \mathrm{fm}^{-2}$ & $2.1 \mathrm{fm}^{-2}$ \\
$\tau=$ & -4.8 & -3.9 \\
$A=$ & $-13.8 \mathrm{MeV}$ & $-11.9 \mathrm{MeV}$ \\
$B=$ & $7.1 \mathrm{MeV}$ & $11.7 \mathrm{MeV}$ \\
$C=$ & 38.3 & 30.8 \\
$D=$ & $-197.3 \mathrm{MeV}$ & $-197.3 \mathrm{MeV}$ \\
$E=$ & $38.5 \mathrm{MeV}$ & $38.5 \mathrm{MeV}$ \\
\hline
\end{tabular}


Table 2. Masses of baryon resonances (all values expressed in MeV) calculated with the Hamiltonian of eq. 9. The column $M_{\text {calc }}^{(\mathrm{I})}$ contains the baryon masses calculated with the set of parameters (Table 1 column (I)) obtained with the analytical fixing procedure (eq. 13) column $M_{\text {calc }}^{(\mathrm{II})}$ contains the baryon masses calculated with the parameters of Table 1 column (II), obtained with a global fit to all the 3 and 4 star experimental data [21].

\begin{tabular}{|c|c|c|c|c|c|}
\hline Baryon & Status & Mass & State & $\mathbf{M}_{\text {calc }}^{(I)}$ & $\mathrm{M}_{\text {calc }}^{(I I)}$ \\
\hline N(938)P11 & $* * * *$ & 938 & ${ }^{2} 8_{1 / 2}\left[56,0^{+}\right]$ & 938.0 & 938.0 \\
\hline N(1440)P11 & $* * * *$ & $1430-1470$ & ${ }^{2} 8_{1 / 2}\left[56,0^{+}\right]$ & 1448.7 & 1455.8 \\
\hline N(1520)D13 & $* * * *$ & $1515-1530$ & ${ }^{2} 8_{3 / 2}\left[70,1^{-}\right]$ & 1543.7 & 1492.9 \\
\hline$N(1535) S 11$ & $* * * *$ & $1520-1555$ & ${ }^{2} 8_{1 / 2}\left[70,1^{-}\right]$ & 1543.7 & 1492.9 \\
\hline$N(1650) S 11$ & $* * * *$ & $1640-1680$ & ${ }^{4} 8_{1 / 2}\left[70,1^{-}\right]$ & 1658.6 & 1585.3 \\
\hline N(1675)D15 & $* * * *$ & $1670-1685$ & ${ }^{4} 8_{5 / 2}\left[70,1^{-}\right]$ & 1658.6 & 1585.3 \\
\hline $\mathrm{N}(1680) \mathrm{F} 15$ & $* * * *$ & $1670-1685$ & ${ }^{2} 8_{5 / 2}\left[56,2^{+}\right]$ & 1651.4 & 1636.6 \\
\hline N(1700)D13 & $* * *$ & $1650-1750$ & ${ }^{4} 8_{3 / 2}\left[70,1^{-}\right]$ & 1658.6 & 1585.3 \\
\hline $\mathrm{N}(1710) \mathrm{P} 11$ & $* * *$ & $1680-1740$ & ${ }^{2} 8_{1 / 2}\left[56,0^{+}\right]$ & 1795.4 & 1760.6 \\
\hline N(1720)P13 & $* * * *$ & $1650-1750$ & ${ }^{2} 8_{3 / 2}\left[56,2^{+}\right]$ & 1651.4 & 1636.6 \\
\hline$\Delta(1232) \mathrm{P} 33$ & $* * * *$ & $1230-1234$ & ${ }^{4} 10_{3 / 2}\left[56,0^{+}\right]$ & 1232.0 & 1251.2 \\
\hline$\Delta(1600) \mathrm{P} 33$ & $* * *$ & $1550-1700$ & ${ }^{4} 10_{3 / 2}\left[56,0^{+}\right]$ & 1683.0 & 1768.9 \\
\hline$\Delta(1620) \mathrm{S} 31$ & $* * * *$ & $1615-1675$ & ${ }^{2} 10_{1 / 2}\left[70,1^{-}\right]$ & 1722.8 & 1713.7 \\
\hline$\Delta(1700) \mathrm{D} 33$ & $* * * *$ & $1670-1770$ & ${ }^{2} 10_{3 / 2}\left[70,1^{-}\right]$ & 1722.8 & 1713.7 \\
\hline$\Delta(1905) \mathrm{F} 35$ & $* * * *$ & $1870-1920$ & ${ }^{4} 10_{5 / 2}\left[56,2^{+}\right]$ & 1945.4 & 1949.7 \\
\hline$\Delta(1910) \mathrm{P} 31$ & $* * * *$ & $1870-1920$ & ${ }^{4} 10_{1 / 2}\left[56,2^{+}\right]$ & 1945.4 & 1949.7 \\
\hline$\Delta(1920) \mathrm{P} 33$ & $* * *$ & $1900-1970$ & ${ }^{4} 10_{3 / 2}\left[56,0^{+}\right]$ & 2089.4 & 2073.8 \\
\hline$\Delta(1950) \mathrm{F} 37$ & $* * * *$ & $1940-1960$ & ${ }^{4} 10_{7 / 2}\left[56,2^{+}\right]$ & 1945.4 & 1949.7 \\
\hline$\Sigma(1193) \mathrm{P} 11$ & $* * * *$ & 1193 & ${ }^{2} 8_{1 / 2}\left[56,0^{+}\right]$ & 1193.0 & 1193.0 \\
\hline$\Sigma(1660) \mathrm{P} 11$ & $* * *$ & $1630-1690$ & ${ }^{2} 8_{1 / 2}\left[56,0^{+}\right]$ & 1703.7 & 1710.7 \\
\hline$\Sigma(1670) \mathrm{D} 13$ & $* * * *$ & $1665-1685$ & ${ }^{2} 8_{3 / 2}\left[70,1^{-}\right]$ & 1798.7 & 1747.9 \\
\hline$\Sigma(1750) \mathrm{S} 11$ & $* * *$ & $1730-1800$ & ${ }^{2} 8_{1 / 2}\left[70,1^{-}\right]$ & 1798.7 & 1747.9 \\
\hline$\Sigma(1775) \mathrm{D} 15$ & $* * * *$ & $1770-1780$ & ${ }^{4} 8_{5 / 2}\left[70,1^{-}\right]$ & 1913.6 & 1840.3 \\
\hline$\Sigma(1915) \mathrm{F} 15$ & $* * * *$ & $1900-1950$ & ${ }^{2} 8_{5 / 2}\left[56,2^{+}\right]$ & 1906.4 & 1891.6 \\
\hline$\Sigma(1940) \mathrm{D} 13$ & $* * *$ & $1900-1950$ & ${ }^{4} 8_{3 / 2}\left[70,1^{-}\right]$ & 1913.6 & 1840.3 \\
\hline$\Sigma^{*}(1385) \mathrm{P} 13$ & $* * * *$ & $1383-1385$ & ${ }^{4} 10_{3 / 2}\left[56,0^{+}\right]$ & 1371.6 & 1390.7 \\
\hline$\Sigma^{*}(2030) \mathrm{F} 17$ & $* * * *$ & $2025-2040$ & ${ }^{4} 10_{7 / 2}\left[56,2^{+}\right]$ & 2085.0 & 2089.2 \\
\hline$\Lambda(1116) \mathrm{P} 01$ & $* * * *$ & 1116 & ${ }^{2} 8_{1 / 2}\left[56,0^{+}\right]$ & 1116.0 & 1116.0 \\
\hline$\Lambda(1600) \mathrm{P} 01$ & $* * *$ & $1560-1700$ & ${ }^{2} 8_{1 / 2}\left[56,0^{+}\right]$ & 1626.7 & 1633.8 \\
\hline$\Lambda(1670) \mathrm{S} 01$ & $* * * *$ & $1660-1680$ & ${ }^{2} 8_{1 / 2}\left[70,1^{-}\right]$ & 1721.7 & 1670.9 \\
\hline$\Lambda(1690) \mathrm{D} 03$ & $* * * *$ & $1685-1690$ & ${ }^{2} 8_{3 / 2}\left[70,1^{-}\right]$ & 1721.7 & 1670.9 \\
\hline$\Lambda(1800) \mathrm{S} 01$ & $* * *$ & $1720-1850$ & ${ }^{4} 8_{1 / 2}\left[70,1^{-}\right]$ & 1836.6 & 1763.3 \\
\hline$\Lambda(1810) \mathrm{P} 01$ & $* * *$ & $1750-1850$ & ${ }^{2} 8_{1 / 2}\left[56,0^{+}\right]$ & 1973.4 & 1938.6 \\
\hline$\Lambda(1820) \mathrm{F} 05$ & $* * * *$ & $1815-1825$ & ${ }^{2} 8_{5 / 2}\left[56,2^{+}\right]$ & 1829.4 & 1814.6 \\
\hline$\Lambda(1830) \mathrm{D} 05$ & $* * * *$ & $1810-1830$ & ${ }^{4} 8_{5 / 2}\left[70,1^{-}\right]$ & 1836.6 & 1763.3 \\
\hline$\Lambda(1890) \mathrm{P} 03$ & $* * * *$ & $1850-1910$ & ${ }^{2} 8_{3 / 2}\left[56,2^{+}\right]$ & 1829.4 & 1814.6 \\
\hline$\Lambda(2110) \mathrm{F} 05$ & $* * * *$ & $2090-2140$ & ${ }^{2} 8_{5 / 2}\left[70,2^{+}\right]$ & 1995.0 & 1957.3 \\
\hline$\Lambda^{*}(1405) \mathrm{S} 01$ & $* * * *$ & $1402-1410$ & ${ }^{2} 1_{1 / 2}\left[70,1^{-}\right]$ & 1657.9 & 1565.6 \\
\hline$\Lambda^{*}(1520) \mathrm{D} 01$ & $* * * *$ & $1518-1520$ & ${ }^{2} 1_{3 / 2}\left[70,1^{-}\right]$ & 1657.9 & 1565.6 \\
\hline$\Xi(1318) \mathrm{P} 11$ & $* * * *$ & 1314-1316 & ${ }^{2} 8_{1 / 2}\left[56,0^{+}\right]$ & 1332.6 & 1332.5 \\
\hline$\Xi(1820) \mathrm{D} 13$ & $* * *$ & $1818-1828$ & ${ }^{2} 8_{3 / 2}\left[70,1^{-}\right]$ & 1938.3 & 1887.4 \\
\hline$\Xi^{*}(1530) \mathrm{P} 11$ & $* * * *$ & $1531-1532$ & ${ }^{4} 10_{3 / 2}\left[56,0^{+}\right]$ & 1511.1 & 1530.2 \\
\hline$\Omega(1672) \mathrm{P} 03$ & $* * * *$ & $1672-1673$ & ${ }^{4} 10_{3 / 2}\left[56,0^{+}\right]$ & 1650.7 & 1669.7 \\
\hline
\end{tabular}

